\title{
Peripheral blood microRNA-15a is a potential biomarker for type 2 diabetes mellitus and pre-diabetes
}

\author{
GHADA AL-KAFAJI $^{1}$, GHAZI AL-MAHROOS ${ }^{2}$, NASREEN A. ALSAYED ${ }^{3}$, \\ ZUHEIR A. HASAN ${ }^{4}$, SADIA NAWAZ ${ }^{1}$ and MOIZ BAKHIET ${ }^{1}$ \\ ${ }^{1}$ Department of Molecular Medicine, College of Medicine and Medical Sciences, Arabian Gulf University, Manama 26671; \\ ${ }^{2}$ Endocrinology Unit, Salmaniya Medical Complex, Salmaniya 13164; ${ }^{3}$ Gulf Diabetes Specialist Centre, Manama 21686; \\ ${ }^{4}$ Department of Physiology, College of Medicine and Medical Sciences, Arabian Gulf University, \\ Manama 26671, Kingdom of Bahrain
}

Received December 25, 2014; Accepted September 2, 2015

DOI: $10.3892 / \mathrm{mmr} .2015 .4416$

\begin{abstract}
MicroRNAs (miRNAs) are small non-coding RNAs that function as crucial regulators of gene expression. Recently, dysregulation of miRNA expression in the blood has been demonstrated to be associated with various diseases, including type 2 diabetes mellitus (T2D), suggesting a potential for their use as biomarkers of disease prognosis. The present study examined the expression levels of T2D-associated miR-15a in peripheral whole blood samples from patients with T2D, pre-diabetes individuals exhibiting impaired fasting glucose (IFG) and impaired glucose tolerance (IGT), as well as healthy control subjects, in order to investigate the potential of peripheral blood miR-15a as a biomarker for the prediction of T2D and pre-diabetes. The present study included 24 patients with T2D, 22 IFG/IGT individuals and 24 healthy controls. The expression levels of miR-15a were analyzed by reverse transcription-quantitative polymerase chain reaction. The results indicated that the peripheral blood miR-15a expression levels were significantly decreased in patients with T2D and IFG/IGT individuals, compared with healthy control subjects $(\mathrm{P}<0.05)$. As determined by multivariate logistic regression analysis, lower miR-15a expression was significantly associated with T2D (odds ratio [OR], 0.51; 95\% confidence interval [CI], 0.16-0.73; $\mathrm{P}<0.05)$ and pre-diabetes (OR, 0.56; 95\% CI, 0.23-0.79; $\mathrm{P}<0.05)$. This association remained statistically significant following adjustment for age, body mass index and hypertension, as well as other biochemical indicators. Furthermore, a receiver operating characteristic analysis revealed that blood miR-15a distinguished patients with T2D
\end{abstract}

Correspondence to: Dr Ghada Al-Kafaji, Department of Molecular Medicine, College of Medicine and Medical Sciences, Arabian Gulf University, 293 Salmaniya Avenue, Manama 26671, Kingdom of Bahrain

E-mail: ghadaa@agu.edu.bh

Key words: type 2 diabetes, pre-diabetes, microRNA, peripheral blood microRNA-15a, biomarker and IFG/IGT individuals from healthy controls (area under the curves; 95\% CI: 0.864 ; 0.751-0.977 and 95\% CI: 0.852 ; $0.752-0.953$, respectively). These results demonstrated that peripheral blood miR-15a expression levels were significantly lower in patients with T2D and IFG/IGT individuals, compared with healthy individuals. Thus, miR-15a in peripheral whole blood may serve as a potential biomarker for T2D and pre-diabetes.

\section{Introduction}

Type 2 diabetes (T2D) is one of the most common chronic diseases worldwide, and it is estimated to affect $>400$ million individuals by 2030 (1). The prevalence of T2D has markedly increased in the states of the Cooperation Council for the Arab States of the Gulf that include Bahrain, and these countries are now among the top 10 with the highest rate of T2D worldwide (2).

A combination of genetic and environmental/epigenetic factors may impair insulin sensitivity in target tissues, as well as insulin secretion from pancreatic $\beta$ cells. When insulin secretion from the $\beta$ cells is no longer able to prevent insulin resistance, glucose levels rise (hyperglycemia), leading to pre-diabetes at an early stage and T2D at a later stage $(3,4)$.

Substantial evidence has demonstrated that there is a long and latent asymptomatic period during which T2D may be detected, and thus T2D may remain undiagnosed for a number of years $(5,6)$. At present, there are no biomarkers for predicting T2D, and the currently available blood glucose tests serve as diagnostic rather than predictive tools. Since lifestyle interventions are highly effective at delaying the onset and progression of the T2D (7), the development of diagnostic or prognostic biomarkers for the early detection of T2D is clinically significant.

MicroRNAs (miRNAs) are endogenous small non-coding RNAs that regulate gene expression by targeting mRNA for cleavage or translational repression. miRNAs are involved in highly regulated processes such as proliferation, differentiation, apoptosis and metabolic processes (8). Specifically, miRNAs are important for $\beta$ cell function and the regulation of glucose stimulated insulin secretion (9-11), and for controlling 
insulin signaling in target tissues (12). Consequently, aberrant expression of several miRNAs has been implicated in the pathogenicity of diabetes (13).

miRNAs have been detected in the peripheral blood in a notably stable form that is protected from endogenous RNase activity (14). The biological function of circulating miRNAs and the exact mechanism underlying the entry of miRNAs into the blood remains elusive. It has been reported that miRNAs may be packaged into microvesicles or associated with apoptotic bodies and actively secreted into the blood circulation, and subsequently target recipient cells in order to exert specific molecular functions, thus mediating cell-to-cell communication $(15,16)$.

To date, changes in single miRNAs and in miRNA signatures have been reported in the serum and plasma of patients with various diseases, such as cancer $(17,18)$ and cardiovascular diseases $(19,20)$. Furthermore, a previous population-based cohort study identified a set of plasma miRNAs that were differentially expressed in patients with T2D and healthy controls (21), and among them, miR-15a was demonstrated to be the most strongly associated with T2D.

In addition to the changes in miRNA expression levels in the serum and plasma, recent blood-based miRNA studies have reported altered miRNA expression in the peripheral whole blood of patients with various types of cancers $(22,23)$, acute myocardial infarction (24), and other human diseases (25), suggesting a potential use of peripheral blood miRNAs as disease biomarkers.

The present study examined the expression levels of T2D-associated miR-15a in peripheral whole blood samples from patients with $\mathrm{T} 2 \mathrm{D}$, pre-diabetes individuals with impaired fasting glucose (IFG) and impaired glucose tolerance (IGT), and healthy control subjects; and investigated the biomarker potential of peripheral blood miR-15a for T2D and pre-diabetes prediction.

\section{Subjects and methods}

Study subjects and clinical data. A total of 70 subjects were included in the present study: 24 patients with T2D (10 men and 14 women), 22 pre-diabetes individuals with IFG and IGT (10 men and 12 women), and 24 healthy control subjects (13 men and 11 women). Both the patients and controls were selected from the King Abdullah University Medical Centre (College of Medicine and Medical Sciences, Arabian Gulf University, Kingdom of Bahrain). Individuals with malignant tumors, cardiovascular disease, nephropathy or other chronic diseases which could effect the miRNA expression levels were excluded from the present study. The study was approved by the Medical Research and Ethics Committee in the College of Medicine and Medical Sciences of the Arabian Gulf University, Kingdom of Bahrain (Manama, Kingdom of Bahrain). Written informed consent was obtained from all subjects.

The presence of T2D in the patients was established according to World Health Organization criteria (26): Fasting glucose $(\mathrm{FG})$ levels $\geq 7.0 \mathrm{mmol} / 1$, glucose levels $\geq 11.1 \mathrm{mmol} / \mathrm{l}(200 \mathrm{mg} / \mathrm{dl})$ as determined by a $2 \mathrm{~h}$ oral glucose tolerance test (OGTT) when glycated hemoglobin (HbAlc) levels $>6.5 \%$, or when the subjects had a clinical diagnosis of the disease. Individuals with FG levels of 6.1-6.9 mmol/1 (110-125 mg/dl) or $2 \mathrm{~h}$ OGTT glucose levels of 7.8-11.0 mmol/1
(140-199 mg/dl) were designated as exhibiting pre-diabetes. The healthy controls were defined as subjects with FG of 4.8-5.2 $\mathrm{mmol} / 1$ (110 mg/dl), and $2 \mathrm{~h}$ OGTT glucose levels of $<7.8 \mathrm{mmol} / \mathrm{l}(140 \mathrm{mg} / \mathrm{dl})$.

miRNA extraction. A total of $5 \mathrm{ml}$ of peripheral blood were collected from the subjects and placed into tubes containing ethylenediaminetetraacetic acid (EDTA; BD Biosciences, Franklin Lakes, NJ, USA). Aliquots ( $0.5 \mathrm{ml}$ each) of blood were mixed with $1.3 \mathrm{ml}$ RNAlater, an RNA stabilization reagent (Ambion Life Technologies, Carlsbad, CA, USA) and stored at $-80^{\circ} \mathrm{C}$. Prior to RNA extraction, the frozen samples were thawed at room temperature, and total RNA including small RNA was isolated using the miRNeasy kit (Qiagen, Inc., Valencia, CA, USA) according to the manufacturer's instructions. Concentration and purity of the RNA was quantified by measuring the absorbance at $260 \mathrm{~nm}$ (A260) and $280 \mathrm{~nm}$ (A280) using a NanoDrop ND-100 spectrophotometer (Thermo Fisher Scientific, Inc., Waltham, MA, USA).

Reverse transcription-quantitative polymerase chain reaction (RT-qPCR). RT-qPCR was performed using the TaqMan ${ }^{\circledR}$ MicroRNA Reverse Transcription kit and Biosystems real-time PCR detection system (Applied Biosystems Life Technologies, Foster City, CA, USA). cDNA was synthesized from total RNA (20 ng) using specific stem-loop RT primers and reagents from the TaqMan ${ }^{\circledR}$ MicroRNA Reverse Transcription kit (3 $\mu$ l specific stem-loop RT primers, $100 \mathrm{mM}$ dNTPs, 10X reverse transcription buffer, $20 \mathrm{U} / \mu 1$ RNase inhibitor, $50 \mathrm{U} / \mathrm{ml}$ MultiScribe $^{\mathrm{TM}}$ Reverse Transcriptase and $4.16 \mu 1$ nuclease-free water to a final volume of $15 \mu \mathrm{l})$.

For qPCR, PCR products were amplified from cDNA using the TaqMan ${ }^{\circledR}$ MicroRNA Assay/TaqMan ${ }^{\circledR}$ Universal PCR Master mix (Applied Biosystems Life Technologies). Briefly, a $1.33 \mu \mathrm{l}$ aliquot of cDNA was combined with $1 \mu \mathrm{l}$ TaqMan miRNA (consisting of the specific PCR primers, and a $20 \mathrm{X}$ TaqMan ${ }^{\circledR}$ MGB probe, TaqMan ${ }^{\circledR}$ Universal PCR Master mix II (2X), no UNG $(10 \mu \mathrm{l})$ and nuclease-free water $(7.67 \mu \mathrm{l})$ to a final volume of $20 \mu \mathrm{l}$. The PCR primers had the following sequences: Mature hsa-miR-15a, UAGCAGCACAUAAUG GUUUGUG; reference gene RNU6B, CGCAAGGATGAC ACGCAAATTCGTGAAGCGTTCCATATTTTT. qPCR was performed using a 7900HT Fast Real Time PCR system (Applied Biosystems Life Technologies) with the following cycling conditions: $95^{\circ} \mathrm{C}$ for $10 \mathrm{~min}$, followed by $95^{\circ} \mathrm{C}$ for $15 \mathrm{sec}$ and $60^{\circ} \mathrm{C}$ for $60 \mathrm{sec}$ for a total of 40 cycles.

The PCR of each sample was run in duplicate to minimize experimental errors. The results were analyzed using Sequence Detection Software version 1.7 (Applied Biosystems Life Technologies). The expression levels of miR-15a were normalized to those of RNU6B, a small nuclear RNA, the expression levels of which were demonstrated to be relatively abundant and constant across a wide range of human tissues and cell line types. It is regarded as one of the control genes with the least variability for miRNAs assays (27) and has been widely used in different fields including in diabetes research $(28,29)$.

The fold change of miR-15a expression was determined using the $2^{-\Delta \Delta \mathrm{Ct}}$ method. The change in threshold cycle $(\Delta \mathrm{Ct})$ was calculated by subtracting the $\mathrm{Ct}$ values of the reference RNU6b from the $\mathrm{Ct}$ values of the target miR-15a. $\Delta \Delta \mathrm{Ct}$ was 
Table I. Characteristics of the subjects.

\begin{tabular}{lccc}
\hline Characteristic & T2D & Pre-diabetes & Controls \\
\hline Number of subjects & 24 & 22 & 24 \\
Age $($ years $)$ & $52 \pm 6.0$ & $49 \pm 7.7$ & $49 \pm 9.1$ \\
Gender $(\mathrm{M} / \mathrm{F})$ & $10 / 14$ & $10 / 12$ & $13 / 11$ \\
BMI $\left(\mathrm{kg} / \mathrm{m}^{2}\right)$ & $25.3 \pm 1.8^{\mathrm{a}}$ & $24.5 \pm 2.2$ & $24.2 \pm 1.0$ \\
Hypertension $[\mathrm{n}(\%)]$ & $14(58.3)^{\mathrm{b}}$ & $9(40.9)^{\mathrm{a}}$ & $1(4.1)$ \\
FG $(\mathrm{mmol} / \mathrm{l})$ & $9.4 \pm 7.6^{\mathrm{b}}$ & $6.4 \pm 5.8^{\mathrm{b}}$ & $4.4 \pm 4$ \\
2 h OGTT $(\mathrm{mmol} / \mathrm{l})$ & $14.6 \pm 2^{\mathrm{b}}$ & $8.8 \pm 0.8$ & $6.1 \pm 0.6$ \\
HbAlc $(\%)$ & $7.5 \pm 0.8^{\mathrm{b}}$ & $6.5 \pm 0.7^{\mathrm{b}}$ & $4.8 \pm 0.6$ \\
TG $(\mathrm{mmol} / \mathrm{l})$ & $2.38(1.0-3.5)^{\mathrm{b}}$ & $2.27(1.0-3.3)^{\mathrm{b}}$ & $1.49(0.9-1.6)$ \\
LDL-C $(\mathrm{mmol} / \mathrm{l})$ & $3.4 \pm 0.5^{\mathrm{b}}$ & $3.17 \pm 0.7$ & $2.99 \pm 0.2$ \\
HDL-C $(\mathrm{mmol} / \mathrm{l})$ & $1.0 \pm 0.1^{\mathrm{b}}$ & $1.29 \pm 0.2$ & $1.31 \pm 0.2$ \\
TC $(\mathrm{mmol} / \mathrm{l})$ & $5.26(4.0-5.8)^{\mathrm{b}}$ & $4.97(3.3-5.5)$ & $4.83(3.8-5.0)$ \\
\hline
\end{tabular}

Data are presented as numbers $(\%)$ for categorical data, mean \pm standard deviation for parametrically distributed data, or median (interquartile range) for non-parametrically distributed data. T2D, type 2 diabetes mellitus; BMI, body mass index; FG, fasting glucose; OGTT, oral glucose tolerance test; HbA1c, glycated hemoglobin; TG, triglyceride; HDL-C, high density lipoprotein cholesterol; LDL-C, low density lipoprotein cholesterol; TC, total cholesterol. ${ }^{\mathrm{a}} \mathrm{P}<0.05$ and ${ }^{\mathrm{b}} \mathrm{P}<0.005$, vs. the controls.

Table II. Association between lower miRNA-15a expression levels and T2D and pre-diabetes.

\begin{tabular}{|c|c|c|c|c|}
\hline \multirow[b]{2}{*}{ miR-15a expression } & \multicolumn{2}{|c|}{$\mathrm{T} 2 \mathrm{D}$} & \multicolumn{2}{|c|}{ Pre-diabetes } \\
\hline & OR $(95 \% \mathrm{CI})$ & P-value & OR $(95 \% \mathrm{CI})$ & P-value \\
\hline Crude & $0.51(0.16-0.73)$ & 0.02 & $0.56(0.23-0.79)$ & 0.01 \\
\hline Adjusted, model $1^{\mathrm{a}}$ & $0.55(0.31-0.82)$ & 0.04 & $0.57(0.28-0.83)$ & 0.05 \\
\hline Adjusted, model $2^{\mathrm{a}}$ & $0.53(0.19-0.79)$ & 0.03 & $0.58(0.37-0.87)$ & 0.03 \\
\hline Adjusted, model $3^{\mathrm{a}}$ & 0.57 (0.37-0.89) & 0.02 & $0.57(0.35-0.80)$ & 0.05 \\
\hline
\end{tabular}

${ }^{a}$ Model 1, adjusted for age and gender. Model 2, adjusted for model 1, hypertension and BMI. Model 3, adjusted for model 2, TG, LDL-C, HDL-C and TC. miRNA, microRNA; T2D, type 2 diabetes; BMI, body mass index; TG, triglyceride; LDL-C, low density lipoprotein cholesterol; HDL-C, high density lipoprotein cholesterol; TC, total cholesterol.

then calculated by subtracting the average $\Delta \mathrm{Ct}$ values of the controls from the average $\Delta \mathrm{Ct}$ values of the cases (diabetes or pre-diabetes).

Statistical analysis. Comparisons between cases and controls were conducted by $\chi^{2}$ or Student's unpaired t-test, and presented as the mean \pm standard deviation (SD). Multivariate logistic regression analysis was used to assess the association between miR-15a expression levels and T2D and pre-diabetes. The area under the curve (AUC) using receiver operating characteristic (ROC) analysis was calculated for miRNA-15a to assess the predictive values. A two-tailed $\mathrm{P}<0.05$ was considered to indicated a statistically significant difference. The data were analyzed using SPSS 19 (IBM, Armonk, NY, USA).

\section{Results}

Characteristics of the study subjects. Patient characteristics are presented in Table I. No significant differences in gender distribution and age were found among T2D patients, pre-diabetes individuals (IFG/IGT), and healthy control subjects $(\mathrm{P}>0.05)$. The body mass index (BMI) was significant higher in patients with T2D, compared with control subjects $(\mathrm{P}>0.05)$, but was not significantly different in individuals with pre-diabetes compared with controls $(\mathrm{P}>0.05)$. The percentage of subjects with hypertension differed significantly between $\mathrm{T} 2 \mathrm{D}$ and pre-diabetes compared with the controls $(\mathrm{P}<0.05)$. In the groups of patients with T2D and pre-diabetes, significant differences were found for levels of fasting glucose, OGTT glucose and HbA1c levels compared with those of the control subjects $(\mathrm{P}<0.005)$. In addition, patients with T2D had statistically different results for levels of triglyceride (TG), high density lipoprotein cholesterol (HDL-C), low density lipoprotein cholesterol (LDL-C) and total cholesterol (TC) compared with controls $(\mathrm{P}<0.005)$. Conversely, pre-diabetes individuals had statistically different results for levels of TG $(\mathrm{P}<0.05)$, but not for HDL-C, LDL-C or TC (P>0.05), compared with the controls. 


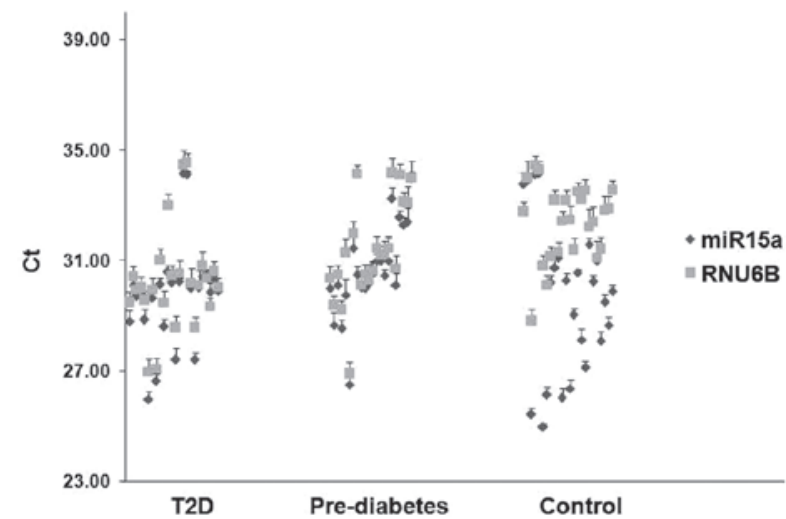

Figure 1. Ct values of miR-15a and RNU6B as determined by RT-qPCR. Total RNA was extracted from the whole blood of patients with T2D $(n=24)$; pre-diabetes individuals $(n=22)$, and healthy control subjects $(n=24)$, and was reverse transcribed to cDNA using miRNA specific primers. RT-qPCR was performed using a TaqMan assay, and the thermal cycle was set to 40 cycles. The $\mathrm{Ct}$ values of miR-15a and RNU6B are presented. Two cDNA replicates were used for each RT-qPCR run. The data are presented as the mean \pm standard deviation. Ct, threshold cycle; RT-qPCR, reverse transcription-quantitative polymerase chain reaction; T2D, type 2 diabetes mellitus; miR, microRNA.

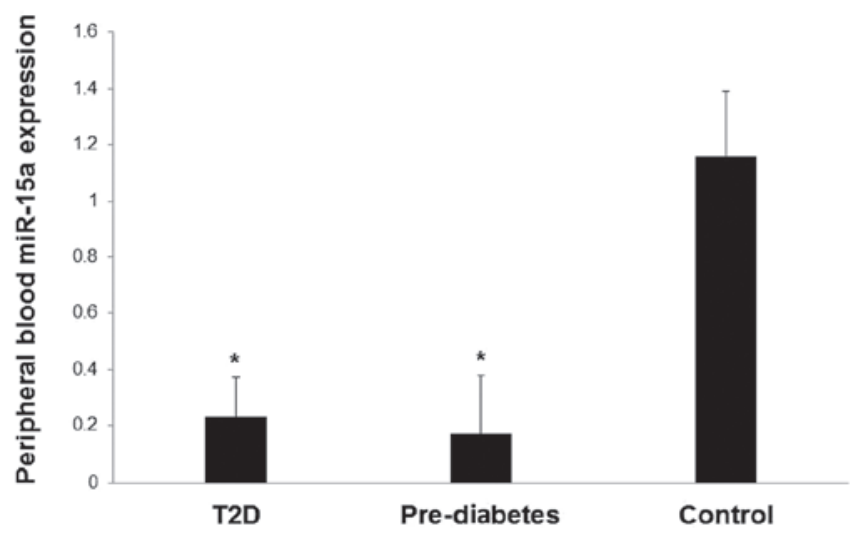

Figure 2. Peripheral blood miR-15a expression levels in patients with T2D, pre-diabetes individuals and healthy controls. Total RNA was extracted from the whole blood of patients with T2D $(n=24)$; individuals with pre-diabetes $(n=22)$ and healthy control subjects $(n=24)$, and was reverse transcribed to cDNA using miRNA specific primers. The expression levels of blood miR-15a relative to RNU6B were determined by reverse transcription-quantitative polymerase chain reaction using a TaqMan assay. The data are shown as the mean \pm standard deviation. ${ }^{*} \mathrm{P}<0.05$, vs. the controls. T2D, type 2 diabetes mellitus; miR, microRNA.

Expression of miR-15a. Using RT-qPCR, the expression levels of miR-15a were determined relative to the endogenous control RNU6B in the peripheral whole blood of patients with T2D, pre-diabetes individuals, and healthy control subjects. miR-15a and RNU6B exhibited reliable $\mathrm{Ct}$ values in all samples from T2D, pre-diabetes and controls. No replicates with a $\mathrm{Ct}$ value $>35$ were detected (Fig. 1).

The expression levels of miR-15a in the blood were significantly lower in patients with $\mathrm{T} 2 \mathrm{D}$ and pre-diabetic individuals compared with healthy controls $(\mathrm{P}<0.05$; Fig. 2$)$. The fold change of miR-15a (calculated as the mean $\pm \mathrm{SD}$ ) was $0.23 \pm 0.14$ for $\mathrm{T} 2 \mathrm{D}, 0.17 \pm 0.21$ for pre-diabetes and $1.16 \pm 0.04$ for healthy controls.
A

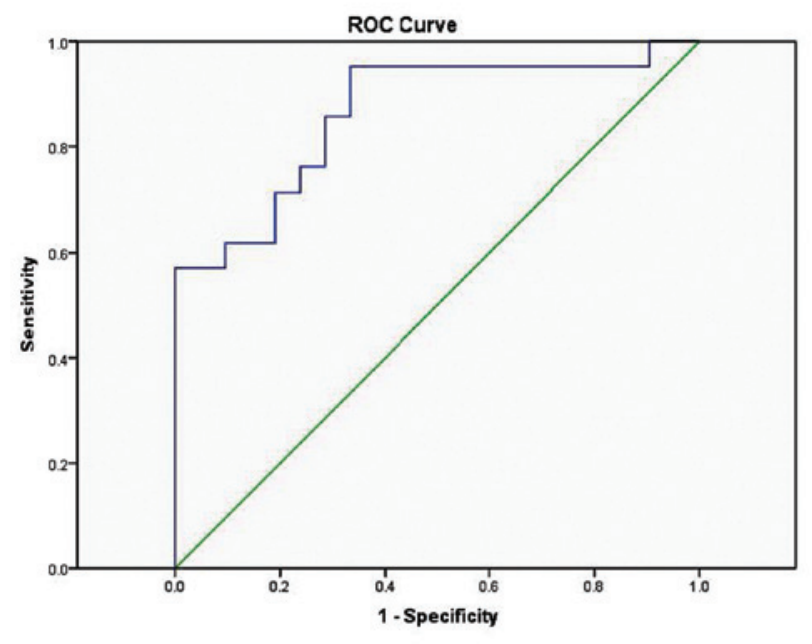

B

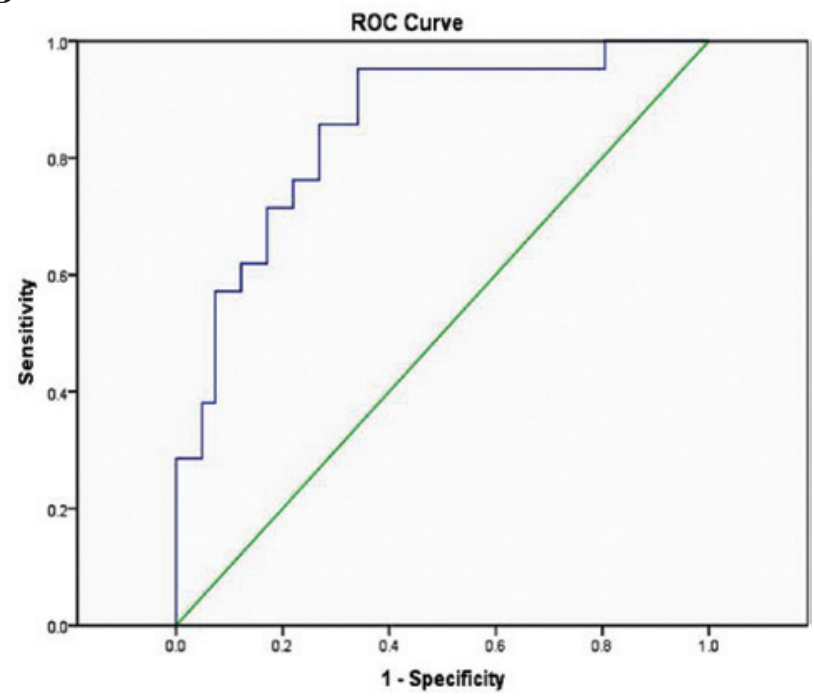

Figure 3. ROC curves of blood miR-15a expression levels. (A) miR-15a expression levels allow distinction between patients with T2D and healthy control subjects (AUC, 0.864; 95\% CI, 0.751-0.977), P $<0.005$. (B) miR-15a expression levels allow distinction between pre-diabetes individuals and healthy controls (AUC, $0.852 ; 95 \% \mathrm{CI}, 0.752-0.953$ ); $\mathrm{P}<0.005$ ). ROC, receiver operating characteristic; AUC, area under the curve; CI, confidence interval; miR, microRNA.

Association between miR-15a and T2D and pre-diabetes. Multivariate logistic regression analysis (Table II) demonstrated strong association between lower miR-15a expression levels and T2D [odds ratio (OR) $0.51 ; 95 \%$ confidence interval (CI) 0.16-0.73; $\mathrm{P}<0.05]$. Similarly, lower miR-15a expression levels were strongly associated with pre-diabetes (OR 0.56; 95\% CI 0.23-0.79; $\mathrm{P}<0.05)$. This association remained statistically significant for $\mathrm{T} 2 \mathrm{D}$ and pre-diabetes following adjustment for age and gender, and further for BMI and hypertension, as well as levels of TG, LDL-C, HDL-C and TC (Table II).

Predictive potential of $m i R-15 a$. To evaluate the predictive potential of blood miR-15a expression levels, ROC analysis was used. The AUC indicated that blood miR-15a expression levels were able to distinguish between patients with T2D and healthy control individuals (AUC 0.864; 95\% CI 0.751-0.977; 
$\mathrm{P}<0.005$ ) (Fig. 3A). Furthermore, blood miR-15a expression levels distinguished individuals with pre-diabetes from healthy controls (AUC 0.852; 95\% CI 0.752-0.953; P<0.005) (Fig. 3B).

\section{Discussion}

Previous studies have reported that serum and plasma miRNAs may serve as novel biomarkers for various diseases, including T2D (14,17-21). Furthermore, miRNAs in peripheral whole blood have also shown promise as a novel class of biomarkers for the detection of several types of cancer, such as brain (22) and breast (23) cancer, as well as acute myocardial infarction (24), and other human diseases (25).

A study by Karolina et al (30) reported an miRNA marker in the peripheral whole blood of patients with T2D patients and IFG. Their study demonstrated that these blood miRNAs (miR-144, miR-146a, miR-150, miR-182, miR-192, miR-29a, miR-30d and miR-320a) may serve as unique biomarkers that are reflective and predictive of T2D (30).

The present study examined the expression levels of T2D-associated miR-15a (21) in the peripheral whole blood of patients with T2D, pre-diabetes individuals (IFG/IGT), and healthy control subjects, and investigated the potential use of blood miR-15a as a biomarker for predicting T2D and pre-diabetes.

The results demonstrated that blood miR-15a expression levels were significantly lower in patients with T2D and IFG/IGT individuals, compared with healthy controls. Furthermore, a strong association was observed between lower miR-15a expression levels and T2D, as well as pre-diabetes. Notably, this association was not altered following adjustment for known risk factors and certain other biochemical indicators.

In a previous population-based cohort study, Zampetaki et al (21) demonstrated a similar pattern of expression, with circulating plasma miR-15a expression levels being lower in patients with T2D. The results from the present study extended the association of lower miR-15a expression levels to pre-diabetes individuals.

A previous study demonstrated that miR-15a is implicated in controlling cell cycle and apoptosis in cancer cells (31). The expression levels of miR-15a were shown to inversely correlate with the expression levels of cyclin D1, and negatively regulate those of B-cell lymphoma 2, a key anti-apoptotic protein (32). Recently, miR-15a has been demonstrated to positively regulate and promote insulin biosynthesis by targeting and inhibiting endogenous uncoupling protein-2 gene expression (33). The overexpression of miR-15a in transfected MIN6 (mouse insulinoma) cells increases insulin secretion, and its suppression decreases insulin levels (33). Therefore, miR-15a was suggested as an important mediator of $\beta$ cell function and insulin synthesis, and a possible therapeutic agent for diabetes (33).

The progression from pre-diabetes (IFG and/or IGT) to early T2D is marked by a decrease in $\beta$ cell function, and thus a decline in insulin secretion, followed by a failure of $\beta$ cells to compensate for insulin resistance with hyperinsulinemia that marks the beginning of T2D $(3,4)$. Individuals with IFG have a 20-30\% chance of developing T2D over the following 5-10 years, and the risk is even higher if individuals with combined IFG and IGT $(34,35)$.
The observation of the present study that blood miR-15a expression levels were decreased in patients with T2D and IFG/IGT individuals may suggest that miR-15a expression correlates with disease development.

In the present study, the ROC curves revealed that blood miR-15a levels have a promising ability to distinguish patients with T2D and IFG/IGT individuals from healthy controls, which suggested a potential use of peripheral blood miR-15 as a biomarker for T2D and pre-diabetes. To the best of our knowledge, the present study demonstrated for the first time that peripheral blood miR-15a may serve as a potential predictive biomarker in T2D and pre-diabetes.

The high stability of miRNAs in human peripheral blood renders them ideal biomarkers for disease detection (14). Given that lifestyle interventions may be highly effective in delaying the onset and progression of T2D (7), the evaluation of peripheral blood biomarkers that are capable of distinguishing between patients with T2D and healthy individuals may lead to a novel preliminary screening method to prevent T2D, through the early identification of individuals who are at a higher risk of developing the disease, particularly those individuals with pre-diabetes.

One of the limitations of the present study was the small sample size, and therefore these results require further investigation in a larger population sample. Additionally, the present study focused exclusively on the association between blood miR-15a expression levels and T2D/pre-diabetes, and did not investigate other miRNAs. Nevertheless, further studies in our laboratory are underway to examine the significance of other whole blood miRNAs as predictive tools for T2D and pre-diabetes. Finally, although the results of the present study demonstrated that blood miR-15a is differentially expressed between patients with T2D, pre-diabetes individuals, and healthy controls, the clinical application of miR-15a in predicting T2D and pre-diabetes requires further investigation and optimization.

In conclusion, the results of the present study demonstrated that the expression levels of miR-15a were significantly lower in the peripheral whole blood of patients with T2D and pre-diabetes individuals, compared with healthy controls, and that miR-15a in peripheral whole blood may serve as a potential biomarker for the prediction of T2D and pre-diabetes.

\section{Acknowledgements}

The authors are grateful to all the participants of the present study, as well as the staff of the Clinical Laboratory of the King Abdullah Medical Centre in the Kingdom of Bahrain. The present study was supported by a research grant from the College of Medicine and Medical Sciences of the Arabian Gulf University, Kingdom of Bahrain (grant no. 81).

\section{References}

1. Wild S, Roglic G, Green A, Sicree R and King H: Global prevalence of diabetes: Estimates for the year 2000 and projections for 2030. Diabetes Care 27: 1047-1053, 2004

2. Alhyas L, McKay A and Majeed A: Prevalence of type 2 diabetes in the states of the Co-operation council for the Arab states of the Gulf: A systematic review. PLoS One 7: e40948, 2012.

3. Abdul-Ghani MA and DeFronzo RA: Pathophysiology of prediabetes. Curr Diab Rep 9: 193-199, 2009. 
4. Cavaghan MK, Ehrmann DA and Polonsky KS: Interactions between insulin resistance and insulin secretion in the development of glucose intolerance. J Clin Invest 106: 329-333, 2000.

5. Harris MI, Klein R, Welborn TA and Knuiman MW: Onset of NIDDM occurs at least 4-7 yr before clinical diagnosis. Diabetes Care 15: 815-825, 1992.

6. Thompson TJ, Engelgau MM, Hegazy M, Ali MA, Sous ES, Badran A and Herman WH: The Onset of NIDDM and its relationship to clinical diagnosis in Egyptian adults. Diabet Med 13: 337-340, 1996.

7. Tuomilehto J, Lindström J, Eriksson JG, Valle TT, Hämäläinen H, Ilanne-Parikka P, Keinänen-Kiukaanniemi S, Laakso M, Louheranta A, Rastas M, et al: Prevention of type 2 diabetes mellitus by changes in lifestyle among subjects with impaired glucose tolerance. N Engl J Med 344: 1343-1350, 2001.

8. Bartel DP: MicroRNAs: Target recognition and regulatory functions. Cell 136: 215-233, 2009.

9. Lynn FC, Skewes-Cox P, Kosaka Y, McManus MT, Harfe BD and German MS: MicroRNA expression is required for pancreatic islet cell genesis in the mouse. Diabetes 56: 2938-2945, 2007.

10. Poy MN, Eliasson L, Krutzfeldt J, Kuwajima S, Ma X Macdonald PE, Pfeffer S, Tuschl T, Rajewasky N, Rorsman P and Soffel M: A pancreatic islet-specific microRNA regulates insulin secretion. Nature 432: 226-230, 2004.

11. He A, Zhu L, Gupta N, Chang Y and Fang F: Overexpression of micro ribonucleic acid 29, highly up-regulated in diabetic rats, leads to insulin resistance in 3T3-L1 adipocytes. Mol Endocrinol 21: 2785-2794, 2007.

12. Plaisance V, Abderrahmani A, Perret-Menoud V, Jacquemin $P$, Lemaigre F and Regazzi R: MicroRNA-9 controls the expression of Granuphilin/Slp4 and the secretory response of insulin-producing cells. J Biol Chem 281: 26932-26942, 2006.

13. Tang X, Tang G and Ozcan S: Role of microRNAs in diabetes. Biochimic Biophysic Acta 1779: 697-701, 2008.

14. Mitchell PS, Parkin RK, Kroh EM, Fritz BR, Wyman SK, Pogosova-Agadjanyan EL, Peterson A, Noteboom J, O'Briant KC, Allen A, et al: Circulating microRNAs as stable blood-based markers for cancer detection. Proc Natl Acad Sci USA 105 10513-10518, 2008.

15. Chen X, Liang H, Zhang J, Zen K and Zhang CY: Secreted microRNAs: A new form of intercellular communication. Trends Cell Biol 22: 125-132, 2012.

16. Kosaka N, Iguchi H, Yoshioka Y, Takeshita F, Matsuki Y and Ochiya T: Secretory mechanisms and intercellular transfer of microRNAs in living cells. J Biol Chem 285: 17442-17452, 2010

17. Chen X, Ba Y, Ma L, Cai X, Yin Y, Wang K, Guo J, Zhang Y, Chen J, Guo X, et al: Characterization of microRNAs in serum: A novel class of biomarkers for diagnosis of cancer and other diseases. Cell Res 18: 997-1006, 2008

18. Yang X, Zeng Z, Hou Y, Yuan T, Gao C, Jia W, Yi X and Liu M: MicroRNA-92a as a potential biomarker in diagnosis of colorectal cancer: A systematic review and meta-analysis. PLoS One 9: e88745, 2014.

19. Wang GK, Zhu JQ, Zhang JT, Li Q, Li Y, He J, Qin YW and Jing Q: Circulating microRNA: A novel potential biomarker for early diagnosis of acute myocardial infarction in humans. Eur Heart J 31: 659-666, 2010.

20. Fichtlscherer S, de Rosa S, Fox H, Schwietz T, Fischer A, Liebetrau C, Weber M, Hamm CW, Röxe T, Müller-Ardogan M, et al: Circulating microRNAs in patients with coronary artery disease. Circ Res 107: 677-684, 2010.

21. Zampetaki A, Kiechl S, Drozdov I, Willeit P, Mayr U, Prokopi M, Mayr A, Weger S, Oberhollenzer F, Bonora E, et al: Plasma microRNA profiling reveals loss of endothelial miR-126 and other microRNAs in type 2 diabetes. Circ Res 107: 810-817, 2010.
22. Roth P, Wischhusen J, Happold C, Chandran PA, Hofer S, Eisele G, Weller M and Keller A: A specific miRNA signature in the peripheral blood of glioblastoma patients. J Neurochem 118: 449-457, 2011.

23. Schrauder MG, Strick R, Schulz-Wendtland R, Strissel PL, Kahmann L, Loehberg CR, Lux MP, Jud SM, Hartmann A, Hein A, et al: Circulating micro-RNAs as potential blood-based markers for early stage breast cancer detection. PLoS One 7: e29770, 2012.

24. Meder B, Keller A, Vogel B, Haas J, Sedaghat-Hamedani F, Kayvanpour E, Just S, Borries A, Rudloff J, Leidinger P, et al: MicroRNA signatures in total peripheral blood as novel biomarkers for acute myocardial infarction. Basic Res Cardiol 106: 13-23, 2011.

25. Keller A, Leidinger P, Bauer A, Elsharawy A, Haas J, Backes C, Wendschlag A, Giese N, Tjaden C, Ott K, et al: Toward the blood-borne miRNome of human diseases. Nat Methods 8: 841-843, 2011.

26. Alberti KG and Zimmet PZ: Definition, diagnosis and classification of diabetes mellitus and its complications. Part 1: Diagnosis and classification of diabetes mellitus provisional report of a WHO consultation. Diabet Med 15: 539-553, 1998.

27. Wong L, Lee K, Russell I and Chen C: Endogenous controls for realtime quantitation of miRNA using TaqMan ${ }^{\circledR}$ MicroRNA assays. Macmillan Publishers Ltd., New York, 2007.

28. Roggli E, Britan A, Gattesco S, Lin-Marq N, Abderrahmani A, Meda P and Regazzil R: Involvement of microRNAs in the cytotoxic effects exerted by proinflammatory cytokines on pancreatic beta-cells. Diabetes 59: 978-986, 2010.

29. Poy MN, Hausser J, Trajkovski M, Braun M, Collins S, Rorsman P, Zavolan M and Stoffel M: miR-375 maintains normal pancreatic alpha- and beta-cell mass. Proc Natl Acad Sci USA 106: 5813-5818, 2009.

30. Karolina DS, Armugam A, Tavintharan S, Wong MT, Lim SC, Sum CF and Jeyaseelan K: MicroRNA 144 impairs insulin signaling by inhibiting the expression of insulin receptor substrate 1 in type 2 diabetes mellitus. PLoS One 6: e22839, 2011.

31. Bandi N, Zbinden S, Gugger M, Arnold M, Kocher V, Hasan L, Kappeler A, Brunnet T and Vassella E: miR-15a and miR-16 are implicated in cell cycle regulation in a Rb-dependent manner and are frequently deleted or down-regulated in non-small cell lung cancer. Cancer Res 69: 5553-5559, 2009.

32. Cimmino A, Calin GA, Fabbri M, Iorio MV, Ferracin M, Shimizu M, Wojcik SE, Aqeilan RI, Zupo S, Dono M, et al: miR-15 and miR-16 induce apoptosis by targeting BCL2. Proc Natl Acad Sci USA 102: 13944-13949, 2005.

33. Sun LL, Jiang BG, Li WT, Zou JJ, Shi YQ and Liu ZM MicroRNA-15a positively regulates insulin synthesis by inhibiting uncoupling protein-2 expression. Diabetes Res Clin Pract 91: 94-100, 2011

34. Meigs JB, Muller DC, Nathan DM, Blake DR and Andres R; Baltimore Longitudinal Study of Aging: The natural history of progression from normal glucose tolerance to type 2 diabetes in the Baltimore longitudinal study of aging. Diabetes 52: 1475-1484, 2003.

35. Bock G, Dalla Man C, Campioni M, Chittilapilly E, Basu R, Toffolo G, Cobelli C and Rizza R: Pathogenesis of pre-diabetes: Mechanisms of fasting and postprandial hyperglycemia in people with impaired fasting glucose and/or impaired glucose tolerance. Diabetes 55: 3536-3549, 2006. 